\title{
THERMODYNAMICS, MNEMONIC MATRICES AND GENERALIZED INVERSES
}

\author{
R. B. LEIPNIK ${ }^{1}$ and C. E. M. PEARCE $₫ 2$
}

(Received 8 August, 2006)

\begin{abstract}
We present an alternative matrix mnemonic for the basic equations of simple thermodynamics. When normalized, this permits an explicit generalized inverse, allowing inversion of the mechanical and chemical thermodynamic equations. As an application, the natural variables $S, V, P$ and $T$ are derived from the four energies $E$ (internal), $F$ (free), $G$ (Gibbs) and $H$ (enthalpy).
\end{abstract}

2000 Mathematics subject classification: primary $80 \mathrm{~A} 10$.

Keywords and phrases: thermodynamics, matrix mnemonics, generalized inverses.

\section{Introduction}

The large number of state variables (eight and more) in simple thermodynamics motivated Born's geometrical mnemonic, the thermodynamic square [2, pp. 117-121]. Koenig [6] elaborated on the idea and provided geometrizations of other thermodynamic relationships. A number of these geometrizations have been drawn together by Fox [4] in the thermodynamic cuboctahedron. Thermodynamic squares have also been assembled in the thermodynamic cube by Pate [9].

In this paper we explore another mnemonic which has been found useful for teaching mechanical and chemical thermodynamics at the University of California at Santa Barbara and which is based on matrix representations. This couples the mnemonics with providing a tool for algebraic analysis. In Sections 4 and 5 we derive mathematical consequences of the mnemonic matrix formulation.

The basic thermodynamic concepts, besides pressure $P$, absolute temperature $T$ and volume $V$ are internal energy $E$, free energy $F$ and entropy $S$. Useful additions

\footnotetext{
${ }^{1}$ (Deceased October 10, 2006), Department of Mathematics, UCSB, Ca 93106-3080, USA

${ }^{2}$ School of Mathematical Sciences, University of Adelaide, Australia 5005; email:

charles.pearce@adelaide.edu.au.

(C) Australian Mathematical Society 2007, Serial-fee code 1446-1811/07
} 
are enthalpy $H$ (in engineering) and Gibbs energy $G$ (in chemical applications). For an account of these see Andrews [1]. Also involved are mole numbers $N_{1}, N_{2}, \ldots$ and their chemical potentials $\mu_{1}, \mu_{2}, \ldots$. It is useful to introduce the four natural variables $[1,2]$ as a column vector $\boldsymbol{Y}=\left[\begin{array}{l}S V P \\ \hline\end{array}\right]^{\prime}$ and the four energies as a column vector $\boldsymbol{A}=\left[\begin{array}{llll}E & H & F & G\end{array}\right]^{\prime}$. Here as subsequently we use boldface letters to denote vectors.

The order of the four energies in $\boldsymbol{A}$ is historical:

(a) internal energy $E$ (Carnot 1824-1828)

(b) enthalpy $H$ (Clausius and Rankine 1850)

(c) free energy $F$ (Kelvin and von Helmholtz 1855)

(d) Gibbs free energy $G$ (Gibbs 1976-1878).

The natural variables $V, P, T$ are pre-scientic. In the gas context, they predate Boyle (1650). Entropy $S$ was introduced by Clausius and Kelvin (1854). The fundamental nature of entropy justifies the term natural.

The order selected for the components of $\boldsymbol{Y}$ is convenient for writing the classic thermodynamic equations

$$
\begin{aligned}
& d E=T d S-P d V \\
& d H=T d S+V d P \\
& d F=-P d V-S d T \\
& d G=V d P-S d T
\end{aligned}
$$

in a convenient matrix form. A matrix form was been pursued earlier by Gilmore [5] but without the mnemonic emphasis. When chemical applications but no other forces, electromagnetic or surface, are introduced, the so-called simple equations [2] are

$$
\begin{aligned}
& d E=T d S-P d V+\mu \cdot d N \\
& d H=T d S+V d P+\mu \cdot d N \\
& d F=-P d V-S d T+\mu \cdot d N \\
& d G=V d P-S d T+\mu \cdot d N
\end{aligned}
$$

with the Gibbs-Duhem relation

$$
0=S d T-V d P+N \cdot d \mu
$$

As may be seen from the above, the natural variables [2] are $S, V, N$ for $E ; S, P, N$ for $H ; T, V, N$ for $F$; and $T, P, N$ for $G$. Equation (1.3) follows from the Eulei relation

$$
E=T S-P V+\mu \cdot N
$$


The letter $E$ is used in place of $U$ for internal energy to keep the energy variables early in the alphabet, the natural variables late and the conversion matrix $M$ in the middle. Later in the paper $U$ is employed for a key intermediary matrix.

We employ also vector dot products such as

$$
\mu \cdot N=\sum_{j=1}^{r} \mu_{j} N_{j} .
$$

Following Callen [2], $N$ is viewed as belonging amongst the natural variables. When there are fixed amounts of a single substance, the convention is to drop $N$ and use the term mechanical or no qualifying adjective with thermodynamics.

The dimension of temperature $T$ is arguable but is usually taken as anstract quantity, degree, written deg. The dimensions of $P, V$ and $E$ are familiar. We have the dimensions

$$
[H]=[F]=[G]=[E] \text { and }[S]=\left[\frac{E}{T}\right]=[E] \mathrm{deg}^{-1} .
$$

Let $P_{0}, V_{0}, E_{0}$ and $T_{0}$ represent unit quantities of $P, V, E$ and $T$ respectively and

$$
\hat{P}=\frac{P}{P_{0}}, \quad \hat{V}=\frac{V}{V_{0}}, \quad \hat{E}=\frac{E}{E_{0}}, \quad \hat{T}=\frac{T}{T_{0}}
$$

the corresponding dimensionless versions. Then also

$$
\hat{H}=\frac{H}{E_{0}}, \quad \hat{F}=\frac{F}{E_{0}}, \quad \hat{G}=\frac{G}{E_{0}} .
$$

Another dimensional convention (see [2]) is to take $[T]=[E]$, but $T_{0} \neq E_{0}$ and $[S]=1$. A third possibility, based on $(1.4)$, is $[S]=[T]=[E]^{1 / 2}$. Leaving the mole numbers alone, it is convenient to write $\hat{\mu}_{j}=\mu_{j} / E_{0}$. To preserve the form of (1.2), (1.3), (1.4), it suffices to take $\hat{S}=S T_{0} / E_{0}$. For the remainder of the paper we omit the hat and take physical quantities as being dimensionless.

In Sections 2 and 3 we present matrix mnemonics for simple mechanical and chemical thermodynamics, giving energies in terms of natural variables via singular matrices. These relations can, however, be solved for the natural variables. The method, originally due to Moore [8] and rediscovered by Penrose [10], employs generalized inverses. We address this problem in Sections 4 and 5 for the mechanical and chemical contexts, respectively.

\section{Mechanical Thermodynamics}

Equation (1.1) may now be represented as

$$
d A=M d Y,
$$


where the "mechanical" matrix $M$ is given by

$$
M=\left[\begin{array}{cccc}
T & -P & 0 & 0 \\
T & 0 & V & 0 \\
0 & -P & 0 & -S \\
0 & 0 & V & -S
\end{array}\right]
$$

This form, which replaces the Born mnemonic, does not appear to have been noticed, even by the early matrix advocate Gilmore [5]. Weinhold [12] used matrices extensively in thermodynamics for computing important partial derivatives, but only invertible matrices. While $M$ is not invertible, it has a Moore-Penrose generalized inverse, which we consider in Section 4.

Regarding mnemonic appeal, the secondary corners of $M$ are zeros and there are two internal zeros on the main diagonal. These connect to form a long hexagonal ring aligned with the cross diagonal. The nonzero entries form a complementary long hexagonal ring aligned with the main diagonal. The determinant det $M$ is zero. The determinant of the upper left $3 \times 3$ minor is $P V T \neq 0$, so $M$ has rank three. By order of column, the nonzero entries are $T,-P, V$ and $-S$, opposite to the order in $Y$. For mnemonic reasons, the column symbols $T, P, V, S$ in (2.2) intercalate with the vowels $\mathrm{O}, \mathrm{A}, \mathrm{E}$ to give the word TOPAVES, to pave the way for system memorization.

\section{Extension to Chemical Thermodynamics}

The same rationale applies to the chemical thermodynamic equations (1.2), except that the Gibbs-Duhem equation (1.3) is appended and a nonzero driving differential five-vector

$$
d R=\left[\begin{array}{c}
e(\mu \cdot d N) \\
-N \cdot d \mu
\end{array}\right]
$$

must be included. Here $e=\left[\begin{array}{llll}1 & 1 & 1 & 1\end{array}\right]^{\prime}$. Put

$$
A^{c h}=\left[\begin{array}{c}
A \\
0
\end{array}\right] \quad \text { and } \quad M^{c h}=\left[\begin{array}{llll} 
& M & \\
0 & 0 & V & -S
\end{array}\right] .
$$

Thus $M^{\text {ch }}$ is $M$ with the fourth row duplicated to make a fifth row, so $M^{c h}$ has rank three. This leads to

$$
d A^{c h}=M^{c h} d \boldsymbol{Y}+d \boldsymbol{R} .
$$

While the form of $d \boldsymbol{R}$ makes (3.1) less attractive than the non-chemical case (2.1). it is still easier than the usual formulation of the chemical case. In the search fo: mnemonics, we note that $d \boldsymbol{R}$ involves the leading letters of "driver", as is appropriate for the driver of the inhomogeneous equation (3.1). This concludes the mnemonics, but as we shall see there are mathematical consequences to the formulation. 


\section{Solutions of the Mechanical Equation}

Although $M$ is singular, (2.1) may be solved for $d Y$ through the Moore-Penrose inverse $\mathrm{M}^{+}$, as the consistency restriction is satisfied in this case. Recall that generally, if $N$ is an $m \times n$ matrix, there is a unique $n \times m$ generalized inverse $N^{+}$satisfying

(a) $N N^{+} N=N$;

(b) $N^{+} N N^{+}=N^{+}$;

(c) $\left(N N^{+}\right)^{\prime}=N N^{+}$;

(d) $\left(N^{+} N\right)^{\prime}=N^{+} N$.

Further

(e) $N \boldsymbol{x}=\boldsymbol{b}$ has infinitely many solutions $\boldsymbol{x}$ provided the consistency condition

$$
N N^{+} \boldsymbol{b}=\boldsymbol{b}
$$

is satisfied. The solutions are defined by

$$
\boldsymbol{x}=N^{+} \boldsymbol{b}+\left(I_{n}-N^{+} N\right) \boldsymbol{q}
$$

for any $n$-vector $q$, where $I_{n}$ is the $n \times n$ identity matrix.

These solutions are of least-squares type, as seen from

(f) If $B$ is a square positive semidefinite matrix, then for each vector $y$ the inequality

$$
(N y-b)^{\prime} B(N y-b) \geq b^{\prime} C b
$$

holds, where

$$
C=B-B N\left(N^{\prime} B N\right)^{+} N^{\prime} B^{\prime} .
$$

If $y$ is of the form

$$
\boldsymbol{y}=\left(N^{\prime} B N\right)^{+} N^{\prime} B \boldsymbol{b}+\left[I_{n}-\left(N^{\prime} B N\right)^{+}\left(N^{\prime} B N\right)\right] \boldsymbol{q},
$$

then (4.1) is an equality. This follows from (e) on replacement of $b$ by $B^{1 / 2} b$ and $N$ by $B^{1 / 2} N$.

With this in hand, consider the problem of forming $M^{+}$. An efficient procedure is the $L U$ method (Strang [11]), a variant of Gauss-Jordan reduction, which will be taken in the $L$-normalized form.

First we obtain the upper triangular matrix

$$
U_{0}=\left[\begin{array}{cccc}
T & -P & 0 & 0 \\
0 & P & V & 0 \\
0 & 0 & V & -S \\
0 & 0 & 0 & 0
\end{array}\right]
$$


by Gauss reduction of $M$. The pivots involved are correctly recycled in the lower triangular matrix

$$
L_{0}=\left[\begin{array}{cccc}
1 & 0 & 0 & 0 \\
1 & 1 & 0 & 0 \\
0 & -1 & 1 & 0 \\
0 & 0 & 1 & 1
\end{array}\right]
$$

so $M=L_{0} U_{0}$. We trim $U_{0}$, by dropping the last row, to

$$
U=\left[\begin{array}{cccc}
T & -P & 0 & 0 \\
0 & P & V & 0 \\
0 & 0 & V & -S
\end{array}\right]
$$

and trim $L_{0}$, by dropping the last column, to

$$
L=\left[\begin{array}{ccc}
1 & 0 & 0 \\
1 & 1 & 0 \\
0 & -1 & 1 \\
0 & 0 & 1
\end{array}\right]
$$

Note that $L U=M$ and that $L$ and $U$ are respectively lower and upper triangular non-square matrices. However $U U^{\prime}$ and $L^{\prime} L$ are square nonsingular matrices.

We now form the left factor $\Phi=U^{\prime}\left(U U^{\prime}\right)^{-1}$ and right factor $\Psi=\left(L^{\prime} L\right)^{-1} L^{\prime}$ of $M^{+}$. Then $M^{+}=\Phi \Psi$ is a $4 \times 4$ matrix satisfying (a)-(d). Explicit expressions are

$$
\Phi=\left[\begin{array}{ccc}
T & 0 & 0 \\
-P & P & 0 \\
0 & V & V \\
0 & 0 & -S
\end{array}\right]\left[\begin{array}{ccc}
T^{2}+P^{2} & -P^{2} & 0 \\
-P^{2} & P^{2}+V^{2} & V^{2} \\
0 & V^{2} & V^{2}+S^{2}
\end{array}\right]^{-1}
$$

and

$$
\Psi=\left[\begin{array}{ccc}
2 & 1 & 0 \\
1 & 2 & -1 \\
0 & -1 & 2
\end{array}\right]^{-1}\left[\begin{array}{cccc}
1 & 1 & 0 & 0 \\
0 & 1 & -1 & 0 \\
0 & 0 & 1 & 1
\end{array}\right]
$$

Finally, on combining the inverses of $\Phi$ and $\Psi$, which both exist when $\boldsymbol{Y} \neq \mathbf{0}$, we derive

$$
M^{+}=\left[\begin{array}{ccc}
T & 0 & 0 \\
-P & P & 0 \\
0 & V & V \\
0 & 0 & -S
\end{array}\right]\left[\begin{array}{ccc}
2 T^{2}+P^{2} & V^{2}-P^{2} & V^{2} \\
T^{2}-P^{2} & V^{2}+P^{2} & V^{2}-S^{2} \\
P^{2} & V^{2}-P^{2} & V^{2}+2 S^{2}
\end{array}\right]^{-1}\left[\begin{array}{cccc}
1 & 1 & 0 & 0 \\
0 & 1 & -1 & 0 \\
0 & 0 & 1 & 1
\end{array}\right]
$$

The loss of symmetry in passing from $M$ to $M^{+}$is striking: in (4.3) $S$ and $T$ appear in only three entries, $P$ and $V$ in eight. An explicit algebraic inverse shows though 
that the determinant of the $3 \times 3$ matrix on the right in (4.3) is positive and symmetric, with value

$$
\left|\begin{array}{ccc}
2 T^{2}+P^{2} & V^{2}-P^{2} & V^{2} \\
T^{2}-P^{2} & V^{2}+P^{2} & V^{2}-S^{2} \\
P^{2} & V^{2}-P^{2} & V^{2}+2 S^{2}
\end{array}\right|=4(P V S T)^{2}\left(P^{-2}+V^{-2}+S^{-2}+T^{-2}\right)
$$

We have that

$$
M M^{+}=L U U^{\prime}\left(L U U^{\prime}\right)^{-1} \Psi=L \Psi=\frac{1}{4}\left[\begin{array}{cccc}
3 & 1 & 1 & -1 \\
1 & 3 & -1 & 1 \\
1 & -1 & 3 & 1 \\
-1 & 1 & 1 & 3
\end{array}\right] .
$$

We readily verify that $M M^{+} \boldsymbol{b}=\boldsymbol{b}$ if and only if the single condition $b_{2}+b_{3}=b_{1}+b_{4}$ is satisfied, a weak consistency condition. If

$$
\begin{aligned}
& \boldsymbol{b}=d A=\left[\begin{array}{ll}
d E d H d F d G & ]^{\prime}, \\
b_{1}+b_{4}-\left(b_{2}+b_{3}\right)=d(E+G)-d(F+H)=0
\end{array}\right.
\end{aligned}
$$

from (1.1), so that consistency is automatically provided.

Hence, in the mechanical case, $d \boldsymbol{A}=M d \boldsymbol{Y}$ has the solutions

$$
d \boldsymbol{Y}=M^{+} d \boldsymbol{A}+\left(I_{4}-M^{+} M\right) d q,
$$

where $d q$ is arbitrary. As may be seen from Equations (4.2) and (4.3), $M^{+}$and $M^{+} M=\Phi \Psi L U=\Phi U$ are interesting but lengthy combinations of the natural variables. The integrated relations

$$
E=T S-P V, \quad H=T S, \quad F=-P V, \quad G=0
$$

in the mechanical case are relevant in calculating $\boldsymbol{A}$ from $\boldsymbol{Y}$, less so for calculating $\boldsymbol{Y}$ from $A$. Thus the inverse route shown in (4.4) is of interest, even though $M^{+}$is so interlocked with $Y$. The problem with (4.5) is that changes in entropy are more easily determined than the full entropy $[3, \mathrm{Ch} . \mathrm{VI}]$.

\section{Solutions of the Chemical Equations}

The procedures of Section 4 for the mechanical equations are directly applicable to the chemical extension. The problem is to solve

$$
M^{c h} d Y=d A^{c h}-d R .
$$


As before

for arbitrary $q$ if

$$
d Y=\left(M^{c h}\right)^{+}\left[d A^{c h}-d R\right]+\left[I_{4}-\left(M^{c h}\right)^{+} M^{c h}\right] d q
$$

$$
M^{c h}\left(M^{c h}\right)^{+}\left[d A^{c h}-d R\right]=d A^{c h}-d R .
$$

We must first calculate the chemical extensions $L^{c h}$ and $U^{c h}$ of $L$ and $U$. Now $U^{c h}=U$, since $M^{c h}$ has the same row vectors as $M$ and so the same $\Phi$. However

$$
L^{c h}=\left[\begin{array}{ccc}
1 & 0 & 0 \\
1 & 1 & 0 \\
0 & -1 & 1 \\
0 & 0 & 1 \\
0 & 0 & 1
\end{array}\right]
$$

and

$$
\Psi=\left[\begin{array}{ccc}
2 & 1 & 0 \\
1 & 2 & -1 \\
0 & -1 & 2
\end{array}\right]^{-1}\left[\begin{array}{ccccc}
1 & 1 & 0 & 0 & 0 \\
0 & 1 & -1 & 0 & 0 \\
0 & 0 & 1 & 1 & 1
\end{array}\right]=\frac{1}{7}\left[\begin{array}{ccccc}
5 & 2 & 2 & -1 & -1 \\
-3 & 3 & -4 & 2 & 2 \\
-1 & 1 & 1 & 3 & 3
\end{array}\right]
$$

Thus

$$
M^{c h}\left(M^{c h}\right)^{+}=L^{c h} \Psi^{c h}=\frac{1}{7}\left[\begin{array}{ccccc}
5 & 2 & 2 & -1 & -1 \\
2 & 5 & -2 & 1 & 1 \\
2 & -2 & 5 & 1 & 1 \\
-1 & 1 & 1 & 3 & 3 \\
-1 & 1 & 1 & 3 & 3
\end{array}\right] \text {. }
$$

The consistency condition $M^{c h}\left(M^{c h}\right)^{+} b^{*}=b^{*}$ for $b^{*}$ reduces to the two conditions

$$
b_{4}^{*}=b_{5}^{*} \quad \text { and } \quad b_{2}^{*}+b_{3}^{*}=b_{1}^{*}+b_{4}^{*} \text {. }
$$

Hence consistency for $\boldsymbol{b}^{*}=d \boldsymbol{A}^{\text {ch }}-\boldsymbol{d} \boldsymbol{R}$ is satisfied if

$$
d H+d F-2 \mu \cdot d N=d E+d G-2 \mu \cdot d N
$$

and

$$
d G=\mu \cdot d N+N \cdot d \mu=d(\mu \cdot N) .
$$

Reference to (1.2), (1.3) shows that both conditions are satisfied, so the chemical case is automatically consistent.

The integrated relations in the chemical case are

$$
\begin{aligned}
& E=T S-P V+\mu \cdot N, \\
& H=T S+\mu \cdot N, \\
& F=\mu \cdot N-P V, \\
& G=\mu \cdot N .
\end{aligned}
$$


The total entropy $S$ and chemical potentials $\mu$ are both difficult to determine [3]. Curiously $F$ is the easiest to calculate from first principles $[2,3,7]$, so $F$ and $S$ provide a simpler route than $\mu$ and $S$ for obtaining other quantities.

\section{References}

[1] F. C. Andrews, Thermodynamics: Principles and Applications (Wiley-Interscience, New York, 1971).

[2] H. B. Callen, Thermodynamics and an Introduction to Thermostatistics (Wiley, New York, 1985).

[3] R. H. Fowler, Statistical Mechanics, 2nd ed. (Cambridge University Press, Cambridge-New York, 1936).

[4] R. F. Fox, "The thermodynamic cuboctahedron model", J. Chem. Educ. 53 (1976) 441-442.

[5] R. Gilmore, "Thermodynamic partial derivatives by matrices", J. Chem. Phys. 75 (1981) 59645966.

[6] F. O. Koenig, "Families of thermodynamic equations I", J. Chem. Phys. 3 (1935) 29-35.

[7] K. C. Lee, "How to teach statistical thermal physics", Amer. J. Phys. 69 (2001) 68-75.

[8] E. H. Moore, "General analysis, part I", Memoirs Amer. Phil. Soc. 1 (1935) 1-231.

[9] S. F. Pate, "The thermodynamic cube", Amer. J. Phys. 67 (1999) 1111-1113.

[10] R. M. Penrose, "A generalized inverse for matrices", Proc. Camb. Phil. Soc. 51 (1955) 406-413.

[11] G. Strang, Linear Algebra and its Applications, 3rd ed. (Harcourt, Brace, Jovanovich, New York, 1988).

[12] F. Weinhold, "Metric geometry of equilibrium thermodynamics I, II, III, IV", J. Chem. Phys. 63 (1975) 2479-2501. 